trueream

* Gated RapidArc

* Five Photon Beam

* High Dose Rate : 2400MU/min

* Supervisor System

* 4D CBCT and Cine Mode

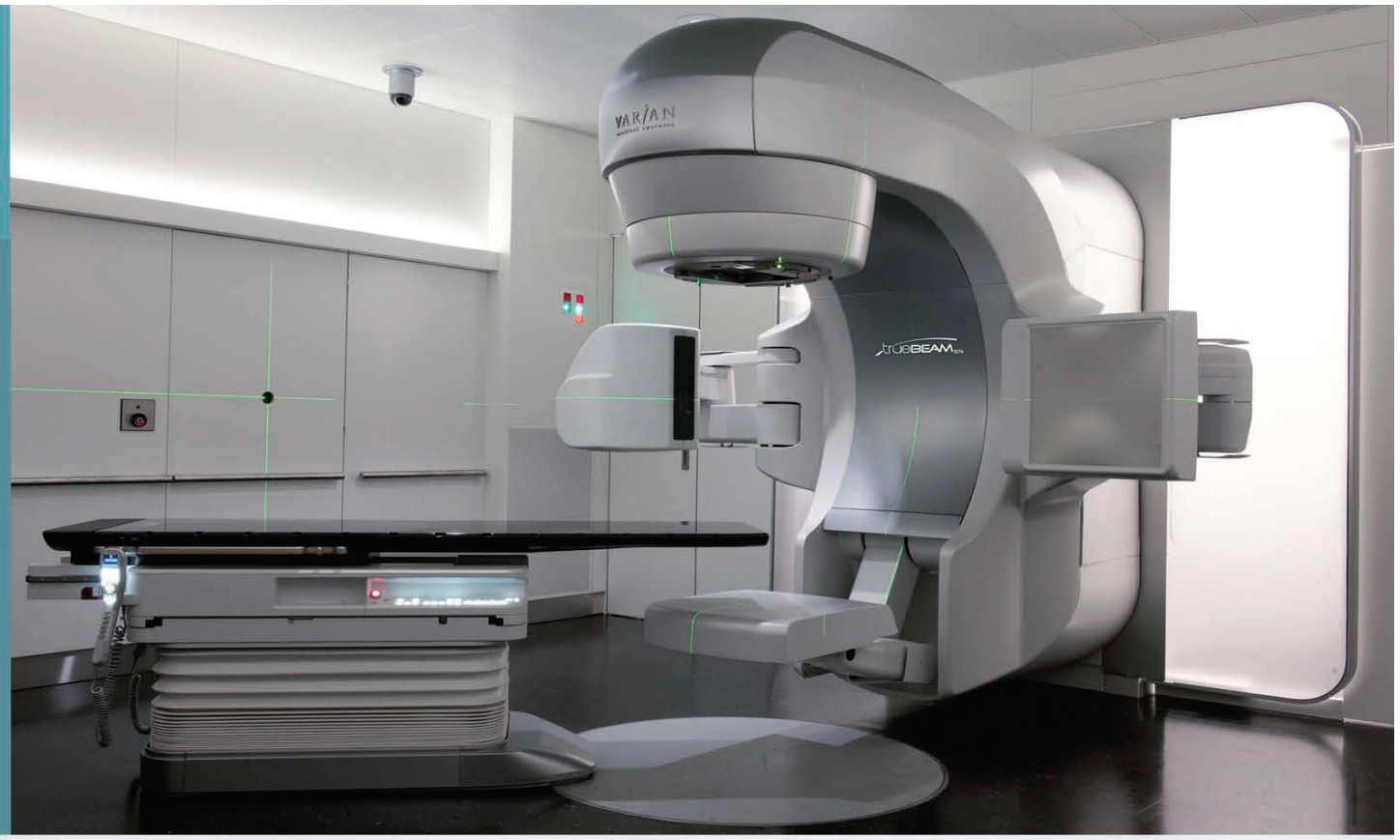

\section{-Viteol $\widehat{E A M}$}

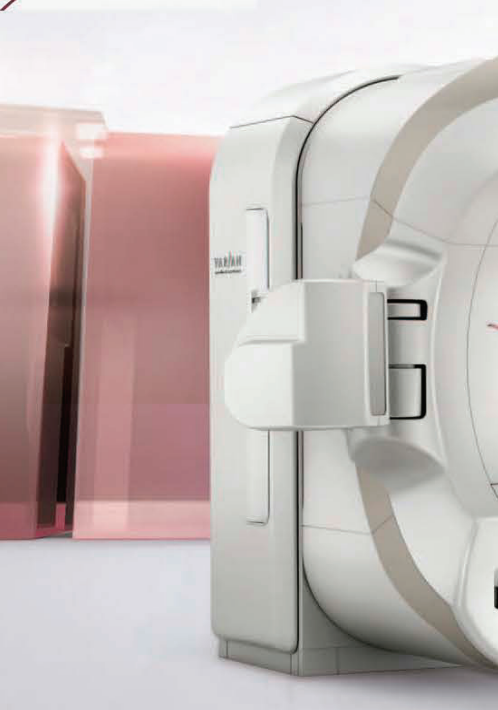

\section{VITAL TOGETHER}

Your oncology expertise combined with the innovative power of VitalBeam can help take your clinic to the next level now and into the future.
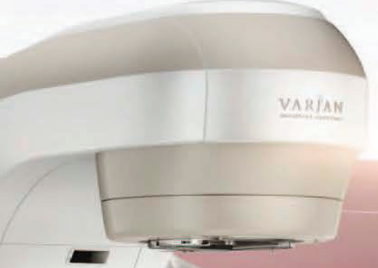

Vitaleman

vilales:aM
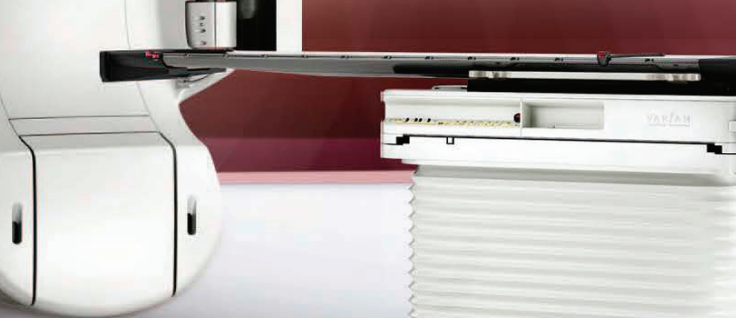
3:
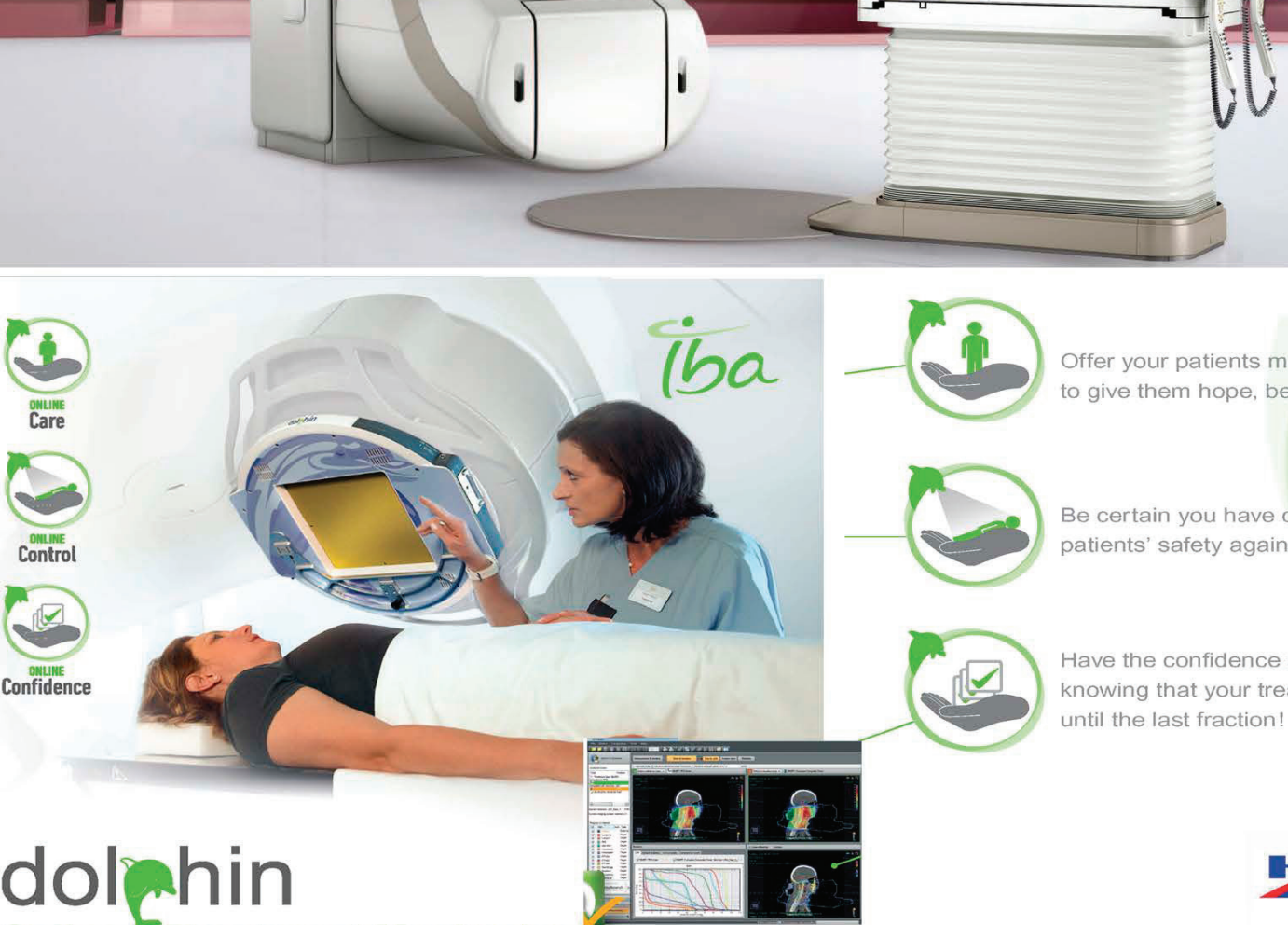

dolahin

Online Treatment Monitoring

\section{- 20}

서울시 종로구 인사동 194-27

TEL : 02) 2003-8441

FAX : 02) $737-4677$ 


\title{
Basic Physical Principles and Clinical Applications of Computed Tomography
}

\author{
Haijo Jung ${ }^{1,2(1)}$ \\ ${ }^{1}$ Development Headquarter, FutureChem Co., Ltd, ${ }^{2}$ Division of Applied RI, Korean Institute of Radiological \& Medical Sciences (KIRAMS), \\ Seoul, Korea
}

Received 28 January 2021

Revised 5 March 2021

Accepted 15 March 2021

Corresponding author

Haijo Jung

(haijo5864@naver.com)

Tel: 82-2-497-3114

Fax: 82-2-497-3115
The evolution of X-ray computed tomography (CT) has been based on the discovery of X-rays, the inception of the Radon transform, and the development of $\mathrm{X}$-ray digital data acquisition systems and computer technology. Unlike conventional X-ray imaging (general radiography), CT reconstructs cross-sectional anatomical images of the internal structures according to $X$-ray attenuation coefficients (approximate tissue density) for almost every region in the body. This article reviews the essential physical principles and technical aspects of the $\mathrm{CT}$ scanner, including several notable evolutions in CT technology that resulted in the emergence of helical, multidetector, cone beam, portable, dual-energy, and phase-contrast $\mathrm{CT}$, in integrated imaging modalities, such as positronemission-tomography-CT and single-photon-emission-computed-tomography-CT, and in clinical applications, including image acquisition parameters, CT angiography, image adjustment, versatile image visualizations, volumetric/surface rendering on a computer workstation, radiation treatment planning, and target localization in radiotherapy. The understanding of $\mathrm{CT}$ characteristics will provide more effective and accurate patient care in the fields of diagnostics and radiotherapy, and can lead to the improvement of image quality and the optimization of exposure doses.

Keywords: Computed tomography, Physical principle, Clinical application, Technical aspects, Radiation dose

\section{Advents of Computed Tomography}

The discovery of X-ray radiation has been a major scientific breakthrough. This has been attributed to Wilhelm Conrad Röentgen (Germany) who conducted the first cathode tube experiments (Crookes tube) in November 8, 1895 [1-3]. This radiation has fluorescence characteristics, sensitizes the film, and penetrates opaque objects. Röentgen called this radiation type $\mathrm{X}$-ray radiation. X-rays are irradiated and penetrate through three-dimensional objects. Xrays which penetrate through objects are recorded on films or detectors as two-dimensional images. This is generally referred to as radiography. Information disappears owing to overlap, tissues with minute absorption coefficients cannot be easily discriminated, and scattering X-rays cause adverse influences on imaging formation.

The term "computed" in CT (computed tomography) indicates calculated or reconstructed, and the term "tomography" is a compound word comprising the term "tomo" (which meaning to "cut" or "section" in Greek) and "graphy" (which means "to describe" in Greek). CT scanner operations are based on X-rays. The typical energy used in gen- 
eral CT is in the range of $100 \mathrm{kV}$ to $150 \mathrm{kV}$.

The algorithms used for CT image reconstruction are based on mathematical foundations of the Radon transformation (Radon theorem) published in 1917 by Johann Radon (Austrian mathematician), who also provided the formula for the inverse transform [3-6]. The Radon theorem states that the image reconstruction is possible from projections: an image distribution function could be obtained from an infinite set of its projections acquired by the rotational scanning.

In the early 1960s, Allan M. Cormack, an American physicist with a South African origin, published the mathematical computation technique based on which the cross-sectional images of internal distributions could be calculated from projection of attenuation data owing to X-rays which penetrated the body at different angles based on the rotation of the X-ray source and electronic detector around a threedimensional object [7-9]. The X-ray-based CT scanner was invented in 1972 by the British engineer Godfrey N. Hounsfield. Hounsfiled shared the Nobel Prize in Physiology and
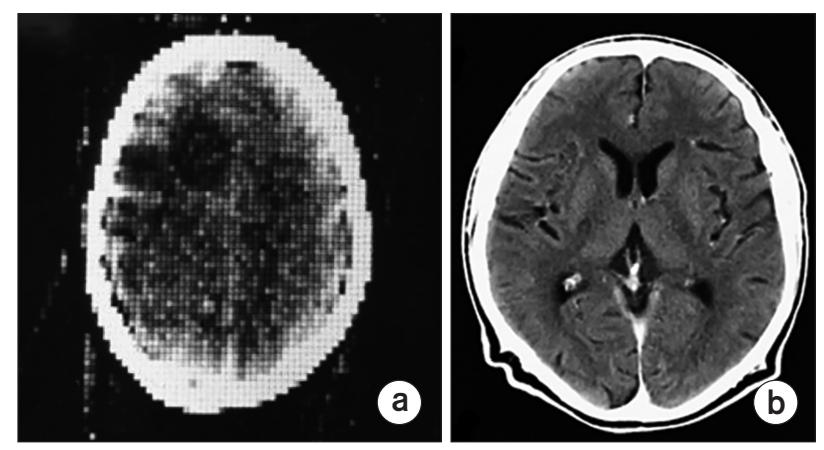

Fig. 1. First clinical computed tomography $(\mathrm{CT})$ image $(80 \times 80$ image matrix obtained from the Atkinson Morley Hospital, London, UK) of the brain acquired in 1971 (a) $[9,10]$, brain CT image acquired recently $(512 \times 512$ image matrix, Siemens SOMATOM Plus 4 [Siemens medical systems, Erlangen, Germany]) with a more advanced CT scanner (b).
Medicine jointly with Allan M. Cormack for the development of CT in 1979. The first clinical CT scanners included a head scanner which was installed at the Atkinson Morley Hospital, London, UK, in 1972, and a whole-body scanner at Georgetown University Medical Center, USA, in 1976. In the early stages of head CT scanners, the image acquisition characteristics were associated with an acquisition time of 7 minutes, an image matrix of $80 \times 80$ pixels, a scan field of $25 \mathrm{~cm}$, and a spatial resolution of $1.3 \mathrm{~mm}(\approx 4$ lines per [lp]/ $\mathrm{cm})$, as shown in Fig. 1 [9,10].

\section{Basic Physical Principles of Computed Tomography}

\section{X-ray attenuation through an object}

The X-rays propagate through the sample wherein some of the X-ray photons are absorbed, and others are transmitted to the detector. The general form of X-ray attenuation is $I_{x}=I_{0} \cdot \exp ^{-\mu x}$, where $I_{0}$ is the X-ray intensity before the object is reached, $I_{x}$ is the X-ray intensity after they penetrate through the object with a thickness $\mathrm{x}$ (where $\exp =\mathrm{e}=$ the exponential coefficient=2.7182818...), $\mu$ is the $\mathrm{X}$-ray linear attenuation coefficient, and $\mathrm{x}$ is the thickness of the absorbing material in chosen distance units, e.g., in mm. If X-rays pass through multiple objects with a total thickness $\mathrm{x}$, as shown in Fig. 2, the penetrated X-ray intensity can be expressed according to $\mathrm{I}=\mathrm{I}_{0} \cdot \mathrm{e}^{-\mu 1 \mathrm{x}} \times \mathrm{e}^{-\mu 2 \mathrm{x}} \times \mathrm{e}^{-\mu 3 \mathrm{x}} \times \mathrm{e}^{-\mu 4 \mathrm{x}}=\mathrm{I}_{0} \cdot \mathrm{e}^{-(\mu 1+\mu 2+\mu 3+\mu 4) \mathrm{x}}$, known as the Lambert-Beer law. Therefore, the total linear attenuation coefficient can be written as the sum of contributions of $\mu_{\mathrm{tot}}=\mu_{1}+\mu_{2}+\mu_{3}+\mu_{4}$ shown in Fig. 2 . Thus, the intensity of the $\mathrm{X}$-rays measured on a position of the detector is proportional to the integral of the two-dimensional (2D) transparency of the object, and is exactly equal to the sum of the linear attenuation coefficient along their passage.

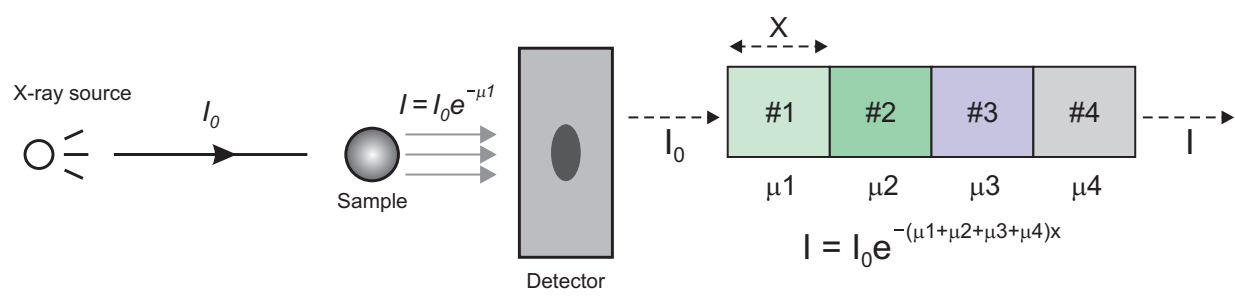

Fig. 2. X-ray beam attenuations passing through of an object (left) and intensity of an X-ray beam passing through an object with multiple different linear attenuation coefficients $\left(\mu_{1}, \mu_{2}, \mu_{3}, \mu_{4}\right)$ (right). 


\section{Computed tomography image data acquisition}

To acquire a CT image, an object is placed on a table. An $\mathrm{X}$-ray source (within the gantry of a CT scanner) rotates around the object, and X-rays which pass through the object are detected on the opposite side. The detections acquired at different angles are sent to the data acquisition system (DAS). These provide projection data to form tomographic images. The basic requirements of CT image acquisition include a) the fact that one tomographic image is reconstructed from X-ray projection data of the object acquired at various angles for general $360^{\circ}$ (or $180^{\circ}$ ) rotations and b) during the scan, objects should be included in every projection dataset, and the object has to be still.

\section{Computed tomography image reconstruction}

Image reconstruction in $\mathrm{CT}$ is a mathematical process that calculates the 2D cross-sectional attenuation distribution function $[\mathrm{f}(\mathrm{x}, \mathrm{y})]$ from a series of one-dimensional (1D) $\mathrm{X}$-ray projections $[\mathrm{P}(\mathrm{r}, \theta) \mathrm{s}]$ acquired as the line integrals at many different angles around the three-dimensional (3D) object, as shown in Fig. 3. A projection, $[\mathrm{P}(\mathrm{r}, \theta)]$, is formed by the attenuated set of parallel X-rays (or isotropic X-rays emitted from a point source) through a $2 \mathrm{D}$ object of interest. A collection of projections at several angles during a single rotation of the X-ray source-detection system is called a sinogram. Effectively, this is a linear transform of the cross-sectional image of the object. Sinograms simply display all of the different projections for any slices stacked

a Simple back projection method
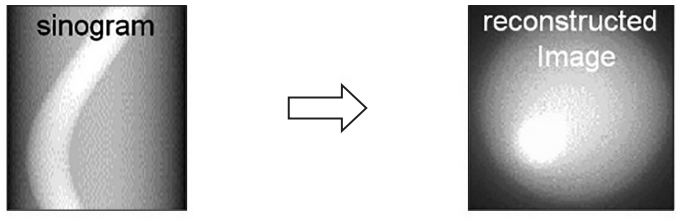

b Filtered back projection method

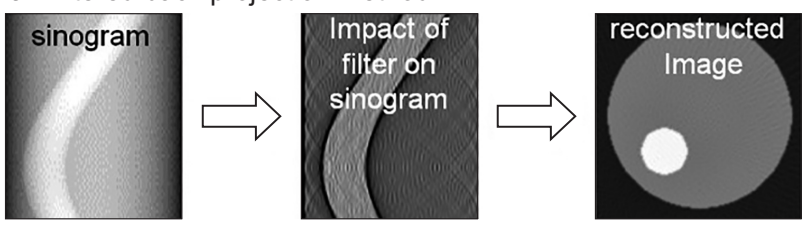

together. In reality, we can know the intensity $\mathrm{I}_{0}$ of the incident X-ray beam and can measure the intensity I of the detected X-ray beam. As a result, what we want to know are the distributions of individual X-ray attenuation coefficients of the object, as shown in Fig. 3. Image reconstruction in CT involves the calculation of these individual coefficients in the internal structure of the object.

There are a lot of methods that can be used for CT image reconstruction: matrix inversion, iterative, backprojection, analytical, 2D Fourier transformation, and filtered backprojection methods in conventional CT; and 3D Radon transform, 3D filtered backprojection methods in spiral CT or conical beam CT (CBCT), and nutating slice. Algorithms that reconstruct tilted image planes are adapted to the spiral path so that the rays are close to the image plane [3,5-7,11-14]. The iterative reconstruction (IR) method is an algebraic reconstruction technique that begins with an image assumption: for a pixelated image value, a single ray in a particular projection is simply the sum of all the pixels the ray passes through on its way through the object, and its value is adjusted based on the difference of estimated and measured values until the two are in agreement. When CT was invented in the 1970s, the original reconstruction algorithm was the IR, which took approximately 45 minutes to reconstruct a single slice. Subsequently, a faster filtered backprojection (FBP) algorithm was introduced, which has been used in CT imaging for more than 30 years. However, the FBP has problems associated with the relatively highnoise levels. The backprojection method inversely "smears back" the projection across the image plane at the measure-

C Fourier transform method

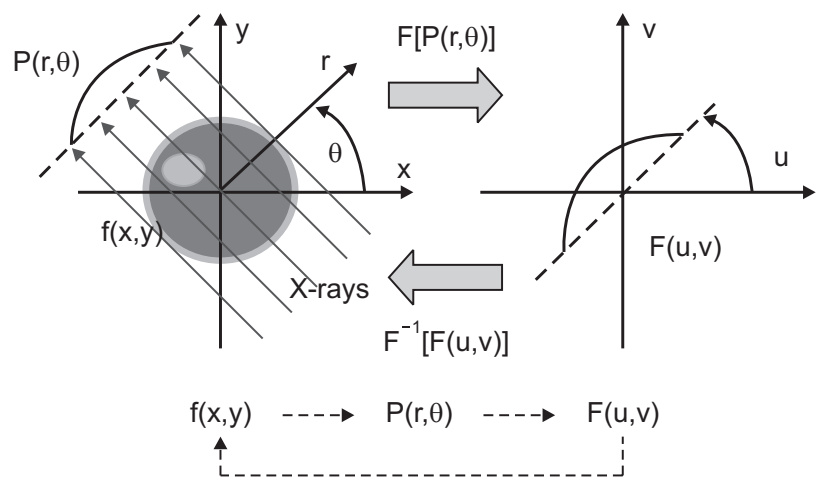

Fig. 3. Computed tomography image reconstruction methods. (a) Simple back projection algorithm method, (b) filtered back projection algorithm method, and (c) Fourier transform algorithm method. 
ment angle for all of the projections. To improve the blurring problem that occurs in backprojection, FBP was introduced with filtering to alter (or modify) the projections with a reconstruction Kernel before standard backprojection was applied. The central slice theorem (CST) (also known as the projection slice theorem or Fourier slice theorem) is the theorem that relates the Radon with the Fourier transform, which is the most common reconstruction method currently used in the medical imaging field.

Our goal is to identify a function $\mathrm{f}(\mathrm{x}, \mathrm{y})$ of the attenuation coefficient map of the object in the $x-y$ rectangular coordinate system from the $\mathrm{P}(\mathrm{r}, \theta)$ function of the projections (sinogram) in polar coordinates. It is always effective to know how various operations in the $\mathrm{f}(\mathrm{x}, \mathrm{y})$ space are related to those in the Fourier F(u,v) space. The CST defines the relationship between the Radon transform of the object and the 2D Fourier transform of the sinogram $\mathrm{P}(\mathrm{r}, \theta)$. As a result, the 1D Fourier transform of the projection measured at an angle $\theta$ is the same as the radial slice acquired through the $2 \mathrm{D}$ Fourier domain of the object at the same angle. Finally, the $\mathrm{f}(\mathrm{x}, \mathrm{y})$ attenuation coefficient distributions are obtained by the inverse Fourier transform of the calculated coefficient distributions of $\mathrm{F}(\mathrm{u}, \mathrm{v})$ in the frequency domain, as shown in Fig. 3.

\section{Computed tomography numbers/Hounsfield units}

The image of a CT scanner is a digital image. It consists of a square matrix of pixels (picture elements), each of which represents a voxel (volume element) of the interior structure of the object (patient). Given that a CT section has a finite thickness, each pixel actually represents a small volume element, or voxel (pixel areaxslice thickness). The size of this voxel depends on the matrix size, the selected fieldof-view (FOV), and the section thickness. These pixel values are the average linear attenuation coefficients of the interior tissue corresponding to the spatial locations of these pixels, and the numerical pixel matrix corresponding to a spatial location in the image is converted into an image based on the allocation/transformation of the pixel values to a corresponding gray scale value.

The typical CT image is constructed on a square matrix of $512 \times 512(262,144$ pixels $)$ and has an image depth of 12 bits
(4,096 gray levels), but has increased to matrices composed of $1,024 \times 1,024$ or $2,046 \times 2,048$ (ultrahigh resolution CT) pixels in recent years. CT numbers (Hounsfield units [HU]) are defined as the attenuation values of the imaged tissues normalized to that of water [15],

$$
\text { CT number }(\mathrm{HU})=1,000 \times \frac{\mu_{\text {pixel }}-\mu_{\text {water }}}{\mu_{\text {water }}},
$$

where $\mu_{\text {water }}$ is the linear attenuation coefficient of water, $\mu_{\text {pixel }}$ is the linear attenuation coefficient of a given pixel. According to the definition of CT number (HU), the CT numbers of air and water range from $-1,000$ and 0 , respectively. The CT numbers for various organs in humans are shown in Fig. 4.

\section{General classifications of computed tomography scanners}

The CT scanner according to the data acquisition method is classified as traditional (axial) and spiral (helical) CT with the fan-beam X-ray source used mainly for diagnostic purposes. In addition, CBCT scanners use the conical beam Xray source mainly for radiation therapy purposes. The conventional (traditional) CT scanner uses a fan-shaped X-ray beam, which takes a single slice image per scan and moves to the next slice scan position. The spiral (helical) CT scanner uses a DAS in which the fan-shaped X-ray beam and detector (or multidetector) move along a helical path relative to the object. In reality, the scanned object on the CT couch is moved to the bore of the scanner while the gantry rotates.

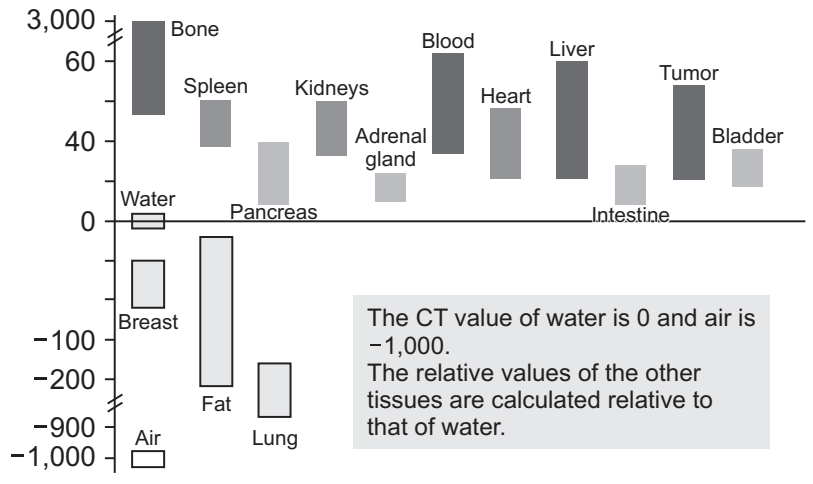

Fig. 4. Hounsfield scale of computed tomography (CT) numbers for various tissues. 
CBCT scanner uses a conical beam X-ray beam which covers a large volume with a single rotation around the scanned object. Conversely, the multidetector CT (MDCT) is equipped with a $2 \mathrm{D}$ array of detector elements which replaces the linear array of detector elements (in a single slice CT) used in conventional and spiral CT scanners. The 2D detector array allows CT scanning to acquire multiple slices (or sections) of large volume simultaneously, and greatly increases the speed of CT image acquisition.

\section{Components of computed tomography scanner}

Basic, modern CT scanners are composed of the gantry equipped with the $\mathrm{X}$-ray imaging acquisition system of $\mathrm{X}$ ray tube, high-voltage generator, filters, collimators, detector arrays, DAS, couch (patient table), operating console, and image reconstruction computer. The modern CT Xray tube has to sustain $60-80$ kilowatts $(\mathrm{kW})$ for up to 20 seconds on a focal spot which is as small as $1.3 \times 10 \mathrm{~mm}$. A highly stable, three-phase generator produces high voltages (generally between 120 to $140 \mathrm{kV}$ ) and supplies it to the Xray tube. The power capacity of the generator listed in $\mathrm{kW}$ determines the range exposure parameters, such as the $\mathrm{X}$ ray characteristics of $\mathrm{kV}$ and $\mathrm{mA}$.

The filter placed between the X-ray tube and the patient plays the role of removing low energy X-rays that do not contribute to image formation but increase patient dose. The collimator is placed between the filter and the patient. Its role reduces the radiation dose and restricts the scattered $\mathrm{X}$-rays from the outer parts of the related slice. Different types of CT detectors were developed, including gas-filled detectors, scintillation detectors (solid-state detectors), and others. A gas-filled detector contains a single vessel filled with high-pressure gases (approximately $25 \mathrm{~atm}$ ) of highatomic number elements (Krypton, Xenon, or Krypton and Xenon) and is divided in separate subdetectors. Early CT scanners used sodium iodide scintillation crystals (NaI) coupled with a photomultiplier tube. Most commonly used solid-state detectors convert the X-rays into visible light photons, which were converted into electrical signals through photodiodes. Solid-state detectors are also called scintillation detectors because of the use of crystals that fluoresce when X-ray photons interact with them. A photo- diode transforms the scintillation lights to electrical signals.

Patient couch (table) is made of carbon fibers to prevent it from interfering with $\mathrm{X}$-rays due to their low-photon absorption properties. Patients lie on the couch and are moved through the gantry aperture to determine the scanning position and control the patients' movements after position settlement during examination. A CT couch should be strong and rigid to support weights up to $204 \mathrm{~kg}$. The shape of the table used in radiation therapy is flat to reconstruct the patient image in the same condition as a treatment machine, while diagnostic CT uses a rounded table top for patient comfort.

The gantry's bore size used in simulations of radiation therapy is bigger to accommodate situations in which the overall diameter of imaging volume is large owing to immobilization devices. Additionally, special patient postures are often needed for better treatments $(85 \mathrm{~cm}$ for radiation treatment vs. $70 \mathrm{~cm}$ for diagnostic purposes). The operating console is the control center of the CT scan. It consists of a keyboard, multiple monitors, and computers which are used to operate the scanner, receive the data from the DAS, and reconstruct the CT image.

\section{Developments of computed tomography scanner}

The first generation of CT scanners [7,16-18] operated as follows. The X-ray pencil beam was measured by a single detector, and the X-ray tube and detector moved together (translation movement). The X-ray and detector then rotated together at a different angle, and these procedures were repeated for a single slice. The X-ray and detector then moved together to a different slice, and the process was then repeated. The second generation CT scanners were operated in the same way as the first generation scanners except for the simultaneous use of a row of up to 30 detectors incorporated with narrow fan X-ray beams. This method required approximately 5-90 seconds per slice to scan. The third generation CT scanner had a row of detectors which was wide enough to image the entire slice at a single angle orientation. Therefore, the translation movement was no longer necessary. The X-ray and detector rotated together at different angles, and this process was repeated until a single slice was scanned before acquisitions were conduct- 
ed for a different slice (axial scanning). This method is most commonly used nowadays, and takes approximately 0.3 seconds to image a single slice. The fourth generation CT adopted a fixed complete ring of detectors. Therefore, the $\mathrm{X}$-ray tube only rotated around a slice and moved to a different slice. This method is not commonly used today. The fifth generation CT electron beam scanners use a stationary $\mathrm{X}$-ray tube and a stationary detector ring, thereby avoiding any mechanically moving parts like the case of the television tube. An electron beam is magnetically deflected onto a fixed array of tungsten anode targets which surround the patient. The magnetic deflection sweeps the electron beam over the target, thus creating an X-ray source that virtually rotates around the patient. Given the absence of mechanically moving parts, a sweep can be accomplished in as little as 50-250 ms [19].

The sixth generation CT, helical (spiral) CT, was developed by Willi A. Kalender (German medical physicist) in 1989. The helical CT combined the priciple of the third and fourth CT generations with slip-ring technology. The patient was moved along the gantry direction through a contiually rotating X-ray beam and detector system in the gantry. Spiral CT is much more efficient in reducing the scanning time. The combination of continuous data acquisition with slip-ring-based volumetric data transmission and continuous table translation led to a considerable reduction of scanning time. The current slip-ring technology of X-ray tube and detector array system installed in the gantry can rotate at rates between 0.25 and 3 seconds per rotation. The seventh generation CT scanners, MDCT, was introduced in the late 1990s and early 2000s with a 2D multiple detector array which simultaneously scanned multiple slices in a single rotation. The development of MDCT has resulted in high-speed image acquisition which is proportional to the number of detector rows. The early, single-slice CT scanner had one row of detectors. However, nowadays all CT scanners are multislice detector arrays and have 8-320 rows of detectors. Multidetector row (or multislice) CT uses multiple rows of CT detectors instead of only one (even single-detector row CT has multiple detectors along the gantry, but only has one row, i.e., it only reconstructs a single slice along the z-direction). The advantage of MDCT is faster scanning, as more parts of the body are contained in each turn of the gantry. For MDCT, multiple slices are reconstructed per projection by the multiple rows of detectors. In MDCT, the pitch is calculated by dividing the table movement by the entire beam width. This yields numbers similar to the single-slice version: a pitch of 1 corresponds to contiguous helices [20].

Today, advanced novel CT technologies introduced dual-energy CT (DECT) and detector-based spectral CT, as shown in Fig. 5. DECT, also known as spectral CT, uses two different X-ray energy tubes (most frequently rated at $80 \mathrm{kVp}$ and $140 \mathrm{kVp}$ ) compared with the general single energy helical CT, thus allowing the image enhancement of substances with the use of subtraction imaging techniques, which have separate attenuation behaviors at different energies. Changing the tube's rated voltage results in materialspecific attenuation changes. DECT exploits the following effects; two X-ray sources running simultaneously at differ-

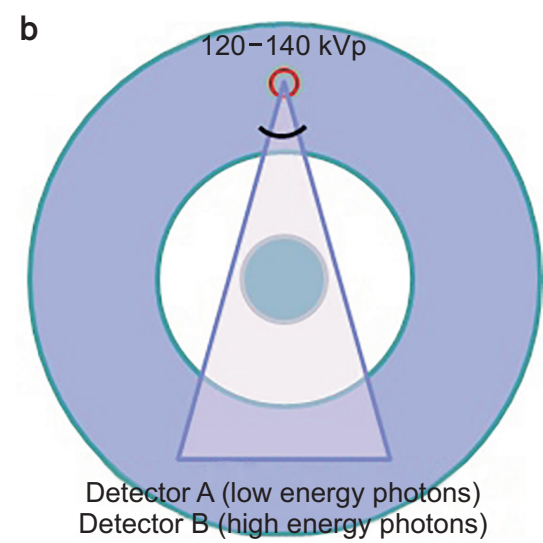

Fig. 5. Structure and mechanism of (a) dual-energy computed tomography (CT) system (adopt two X-ray tubes and corresponding detector arrays) and (b) detector-based spectral CT system (single X-ray tube and two layers within detector arrays). 
ent energies acquire two datasets showing different attenuation levels. In the resulting images, the material-specific difference in attenuation enables an easy classification of the elementary chemical composition of the scanned tissue. In addition, a fused image is provided for initial diagnosis. DECT techniques may be useful to differentiate substances such as iodine, calcium, and uric acid from soft tissues [2124]. Detector-based spectral computed tomography employs dual layers of detectors to simultaneously collect low and high-energy data generated from a single X-ray tube in all patients using conventional helical CT protocols. This spectral information is able to display the varying shades of gray or even colors in the final image to distinguish the $\mathrm{X}$ ray attenuation differences of various elements in the body based on their material densities or atomic numbers. DECT can already facilitate better discrimination of tissues, thus making it easier to differentiate between materials, such as tissues containing calcium and iodine that can appear similar on traditional, monochromatic CT techniques. It can also potentially increase diagnostic accuracy in a wide range of clinical applications, such as the enhancement of the conspicuity and detection of smaller vessels associated with subsegmental pulmonary emboli $[25,26]$.

The research and development of CT technology applications targeted the fields of dental CT and microCT (or small animal) after 2000. The developments of microCT systems for small animal imaging were published in the early $2000 \mathrm{~s}$ $[27,28]$. Findings were commercialized to the industrial, medical, and preclinical microCTs by NanoFocusRay Co.,
Ltd. (Seoul, Korea), as shown in Fig. 6a. The development of domestic, commercial dental CT was led by Vatech Co., Ltd. (Seoul, Korea), as indicated in Fig. 6b, and by Raymedical Co. (Seoul, Korea), Ltd, HDXWill (Seoul, Korea), and others, since the early to mid-2000s. The Health and Medical Division of Samsung Electronics Co. Ltd., (Suwon, Korea) introduced its NExCT 7 CT (stationary) scanner in collaboration with NeuroLogica Corporation (Danvers, MA, USA). This system has a detector coverage of $80 \mathrm{~cm}$ with 128 detector rows, a speed of 0.25 seconds per rotation, a $78 \mathrm{~cm}$ aperture, and a $50 \mathrm{~cm}$ FOV, as demonstrated at the Radiological Society of North America (RSNA) Conference in Chicago in 2015 (see Fig. 6c). Additionally, Samsung Electronics unveiled a mobile CT OmniTom (specifications included 16 slices with a thickness of $0.625 \mathrm{~mm}$, a gantry size of $40 \mathrm{~cm}$ for the coverage of the adult head and neck, and full body scanning of pediatric patients which is ideal for cranial examination procedures) at the RSNA in 2017.

\section{Clinical Applications of Computed Tomography}

\section{Computed tomography scanning parameters}

Parameters responsible for the specifications and performances of X-ray CT scanners are closely related to each other, and are various. The CT scanning time generally depends on the type of examination and the performance specifications of CT scanner. Short scan times are required
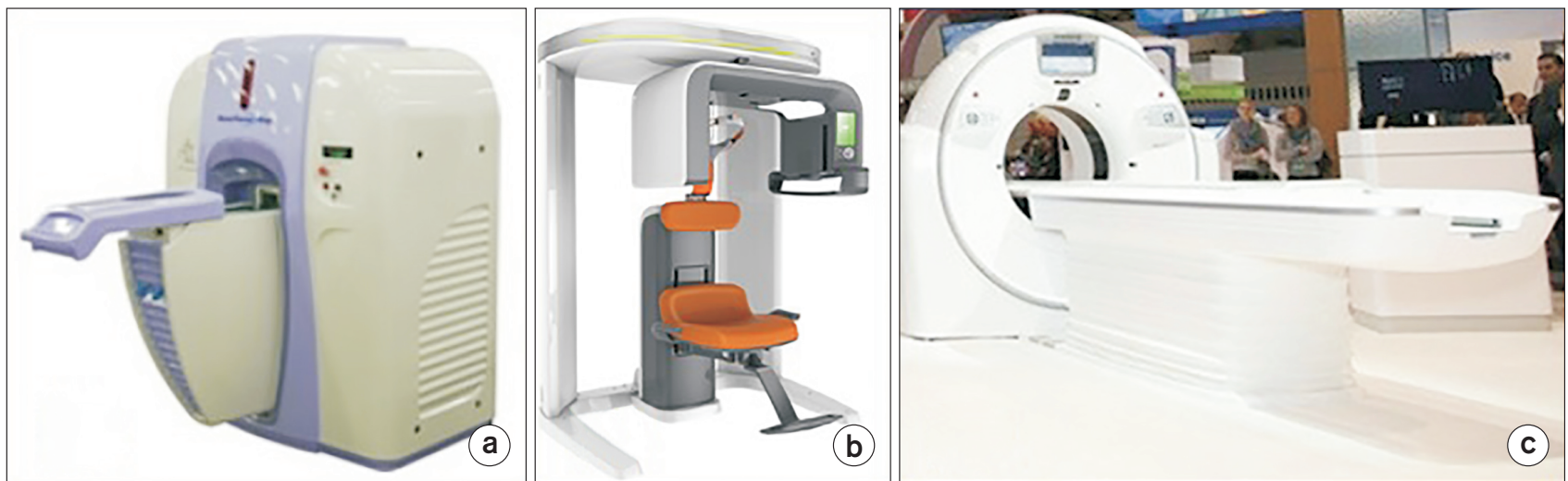

Fig. 6. Domestic commercial computed tomography (CT) scanners. (a) NanoFocusRay's gantry of the rotational microct system (NanoFocusRay Co., Ltd., Seoul, Korea; 2008). (b) Vatech's conical beam dental CT (PaX-13D Green Premium) (Vatech Co., Ltd., Seoul, Korea; 2012). (c) Diagnostic CT Scanner (NExCT 7) (Samsung Electronics Co. Ltd., Suwon, Korea; 2015). 
to reduce the artifacts attributed to the patient's physiological motions, including the respiratory, digestive tract, peristaltic, and others. Gantry rotation time for a helical CT is defined as the amount of time required to complete one full rotation $\left(360^{\circ}\right)$ of the $\mathrm{X}$-ray tube and detector around the object. The advances in technology have shortened the gantry rotation time to values as low as approximately 0.3 seconds. The common CT examination requires a scan length of $240 \mathrm{~mm}$ for the head and $400 \mathrm{~mm}$ for the chest or abdomen. In general, CT slice thickness (slice collimation) is typically $5 \mathrm{~mm}$ for a standard head CT scan, and ranges between 0.625 and $1.25 \mathrm{~mm}$ for both blood vessel and thinsection facial bone CT, or 3D image processing. CT patient throughput is related to the number of examinations per hour, and indicates the ability of scan processing.

\section{Causes and types of computed tomography image artifacts}

Fundamentally, image quality in CT, as in all other medical imaging modalities, is described based on spatial resolution, image contrast, image noise, and artifacts, which are closely related to each other. CT image quality is influenced by the imaging system (technical parameters), user (radiology technologist or radiologist during image acquisition and evaluation), and patient (patient's movement and size). Artifacts indicate features that appear in CT images that did not exist in the original clinic. This primary reason is attributed to the principal limitation of CT and the malfunction and maladjustment of CT itself.

There are many different types of artifacts that originate from motion, metal, partial volume, beam hardening, technical defects, or operator errors. Motion artifacts are patient-based features produced by respiratory, muscular, or other movement by the patient during a scan. If patients move, motion artifacts can arise. Therefore, patient movements should be minimized to prevent these. Motion artifact can be minimized by using a motion artifact correction algorithm together with the control of breathing during image acquisition. Additionally, motion artifacts can be compensated for by the motion correction algorithm. Partial volume artifacts occur when tissues with considerably different X-ray absorption properties in complicated bone structures are included in the same CT slice. These result in a beam attenuation, which is proportional to the average value of each slice. This is because the very dense structures (bones) are only partially included in the slice, and thus result in high-contrast errors. These are attributed to the partial volume effect in the case of large structural variations toward the slice thickness direction. To reduce the partial volume artifacts, the use of a thin-slice thickness is recommended. Beam hardening is the phenomenon that occurs when an X-ray beam composed of different energies passes through a dense object, thus resulting in the selective attenuation of lower energy photons. The beam hardening effect is conceptually similar to a high-pass filter in that only higher energy photons are left to contribute to the beam. Thus, the mean beam energy is increased ("hardened"). The beam hardening effect gives rise to streaking (dark band) artifacts, which appear as multiple streaking bands positioned between two dense tissues or cupping artifacts, thus causing the edges of an object to appear brighter than the center. To effectively reduce the beam hardening effect, the X-ray spectrum has to minimize lower energy photons by adopting a metal filter before the CT scan. In this respect, CT scanners are equipped with metal artifact reduction algorithms. Metal streak artifacts are caused by multiple mechanisms, including beam hardening, scatter, Poisson's noise, motion, and edge effects in the scanned objects, including hair pins, clips, and metals inside the patient. Metals, such as gold-implanted objects within a human body absorb the X-ray beam almost completely, thus producing "radiation shadows" which generate pronounced dark and bright streak artifacts over the entire reconstructed image. This can only be avoided via a gantry tilt that excludes the disturbing metallic objects from the slice plane. Ring artifacts occur owing to the miscalibration or technical failure of one or more detector elements in a CT scanner. This is also caused by the insufficient X-ray intensity or the contamination of a contrast medium on the detector cover. They occur close to the isocenter of the scan and are usually visible on multiple slices at the same location. The ring artifacts appear as concentric rings superimposed on CT images. The ring artifact effects can be remedied by recalibrating the scanner's malfunction detector system or replacing the malfunction detector elements. 


\section{Computed tomography image noise}

Image quality has many components and is influenced by many technical parameters. Noise in CT reduces the visibility of low-contrast objects as unwanted changes in pixel values in a homogeneous image. Noise affecting contrast resolution depends on $\mathrm{mAs}, \mathrm{kVp}$, reconstruction algorithm, slice thickness, patient size, operating mode, image display, etc.

Image reconstruction algorithms depend on the noise levels of CT images. An edge-enhancing (HighRes) algorithm produces good edge definition, but also has highnoise levels. HighRes algorithms provide better spatial resolution but generate more noise. A smoothing algorithm produces a low noise level, but also poorer edge definition. Soft algorithms provide better contrast detectability with less noise. For routine studies, a standard algorithm is normally recommended.

Image noise is inversely related to the standard deviation of the beam thickness. Thinner slices increase image noise; however, the visibility of small lesions is improved. Thicker slices generate less noise and better contrast detectability for soft tissues. Thinner slices generate better spatial resolution for bony structures. Therefore, a balance between contrast detectability and the spatial resolution for the diagnostic content should be considered as a function of slice thickness.

For bigger patient body sizes, the noise level doubles every $10 \mathrm{~cm}$ increase in patient diameter in abdominal scans, and every $13 \mathrm{~cm}$ increases in lung scans according to generally accepted guidelines. If the dose (proportional to
$\mathrm{mAs}$ ) is doubled, the noise level is reduced by a factor of 1.4 (square root of 2). In the case of soft tissue studies, it is most important to keep noise to a minimum by using higher $\mathrm{mAs}$. The lower the noise level is, the easier it is to recognize structures with minute differences in density. However, for bone or lung studies, higher mAs are not necessary [29].

\section{Computed tomography image reformatting}

The most common application of X-ray CT refers to crosssectional (axial) images, which are used for diagnostic and therapeutic purposes in various medical fields. The modern CT workstations are specifically designed for navigation and visualization of various reformatted images, such as the techniques of multiplanar reconstruction (MPR), maximum intensity projection (MIP), minimum intensity projection (MinIP), cine (movie) mode, shaded surface display (SSD, also known as surface rendering), 3D volume rendering, and virtual endoscopy (VE), such as angioscopy, bronchoscopy, and colonoscopy [30].

The MPR technique involves the image processing tasks needed to convert thin-slice data from volumetric CT in the axial plane from another plane (such as coronal, sagittal, or oblique) to control the window level (WW) and center the image to view whichever structures needed with the aid of various software and a workstation, as shown in Fig. 7. From the 3D reconstruction of a sequence of tomographic images, the MPR display can be used to generate interactive slices in Cartesian planes (axial, sagittal, and coronal), or even in oblique planes. This means that the physician can obtain views of the patient's internal structures that provide
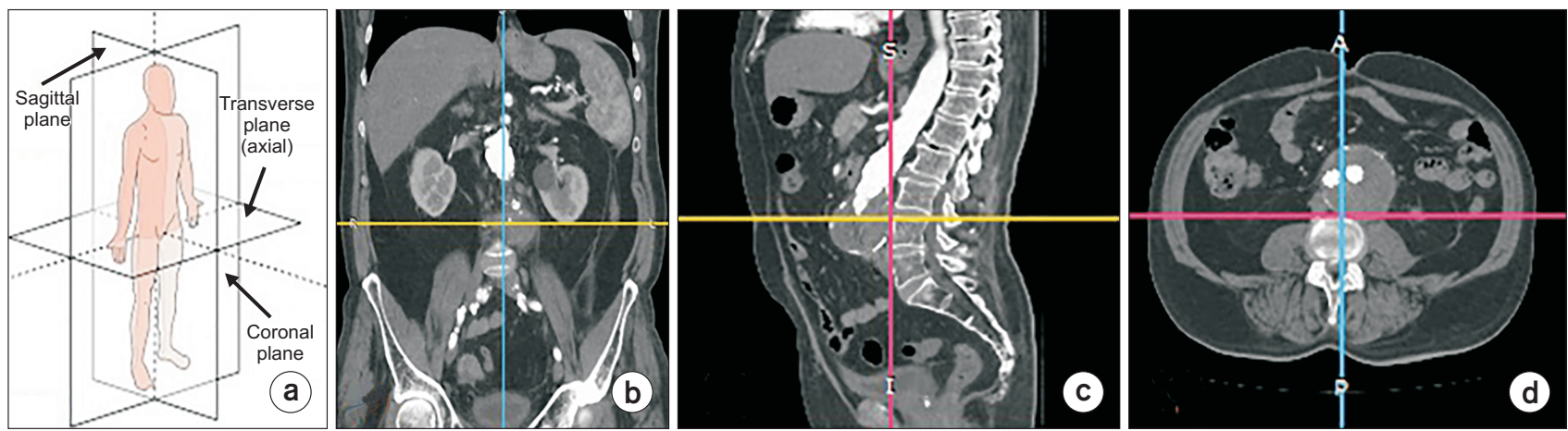

Fig. 7. (a) Anatomical planes in human, multiplanar reconstruction computed tomography images, such as (b) coronal and (c) sagittal converted from (d) axial. 
greater clarification compared with the images from the original sequence.

The MIP technique is a method for 3D data that projects the voxels with the maximum intensity (highest Hounsfield number) that fall in the way of parallel rays traced from the viewpoint to the plane of projection in the $2 \mathrm{D}$ viewing plane to identify all the hyperdense structures (bone or contrast material-filled structures) in a volume. The MinIP technique is a $2 \mathrm{D}$ visualization method that enables detection of low-density structures in a specific volume. The MinIP images, mainly used to diagnose lung diseases, are multiplanar slab images produced by displaying only the lowest attenuation value encountered along a ray cast through an object toward the viewing plane. The surface shaded display represents a visualization technique, which is well established for $3 \mathrm{D}$ imaging of sectional image data by using edge detection image processing algorithms. A SSD provides a realistic 3D view of the surface of a structure of interest within the specified volume by selecting attenuation thresholds. The volume rendering is a type of data visualization technique, which creates a $2 \mathrm{D}$ projection from $3 \mathrm{D}$ imaging of sectional image data by means of a specified software. In volume rendering, transparency, color, and shading are used to allow a better visualization of the volume to be shown in a single image. Volume rendering is primarily conducted for better visualization of the human anatomy, surgical treatment planning, as well as for medical teaching [31]. VE is a noninvasive technique, which combines the features of endoscopic viewing to explore internal structures and cross-sectional volumetric imaging, thus providing precise spatial relationships of pathological regions and their surrounding structures. VE has been used to evaluate the colon, bronchi, stomach, blood vessels, bladder, kidney, esophagus, etc., inside the human body [32].

The CINE mode is a series of rapidly recorded images acquired at sequential cycles of time and displayed on a monitor in a dynamic movie display format. CINE CT images are acquired with four-dimensional (4D) helical CT in CINE mode. A stationary volume scanning mode in planar geometry is being consecutively scanned in space and continually in time. Real-time continuous observations have become available during scans like X-ray fluoroscopy ow- ing to the realization of high-speed image reconstruction. The CINE CT images are sequentially displayed continuous slice images on a display device with $3 \mathrm{D}$ data like a movie. It is easy to distinguish differences between each image in the stack, and to observe the shapes of organs and the function of blood vessels because these images are continually reviewed.

\section{Computed tomography image windowing}

CT images can be displayed with arbitrary brightness and contrast by adjusting the WL and window width (WW), as shown in Fig. 8. The WL is the position describing the center of the scale, which determines the brightness. The WW is the range of CT numbers selected for grayscale amplification, which determines the contrast. The range of CT density values ranges from $-1,000$ to $+3,000$, but the human eye can distinguish only 30-40 grayscale levels at best. Thus, the window settings must be in accordance with the structures to be visualized. This widowing process will change the appearance of the image to highlight particular structures in anatomical or pathological features, as shown in Fig. 8.

A narrow WW indicates that the transition from black to white will take place much faster, which appears as a highcontrast image, but structures outside that window range may be inadequately represented or overlooked. Narrow WWs (defined as the range from 50 to $350 \mathrm{HUs}$ ) is effective for displaying tissue types with similar tissue densities, such as the brain or liver. A wide WW (defined as the range of 400 to 2,000 HUs) indicates that there is a broader grayscale, thus indicating that minor density differences appear homogeneous, such as the lungs or cortical tissue. If the goal is to evaluate all tissues on each image, a wide $\mathrm{WW}$ is effective for displaying tissue types that rapidly vary in image densities, as shown in Fig. 9.

\section{Clinical challenges of computed tomography scanner technology}

CT Angiography (CTA) $[17,33,34]$ is a type of medical examination that combines a CT scan with an injection of a special contrast media (dye) to enhance the signal intensity from arteries, veins, and tissues throughout the body. CTA 
a

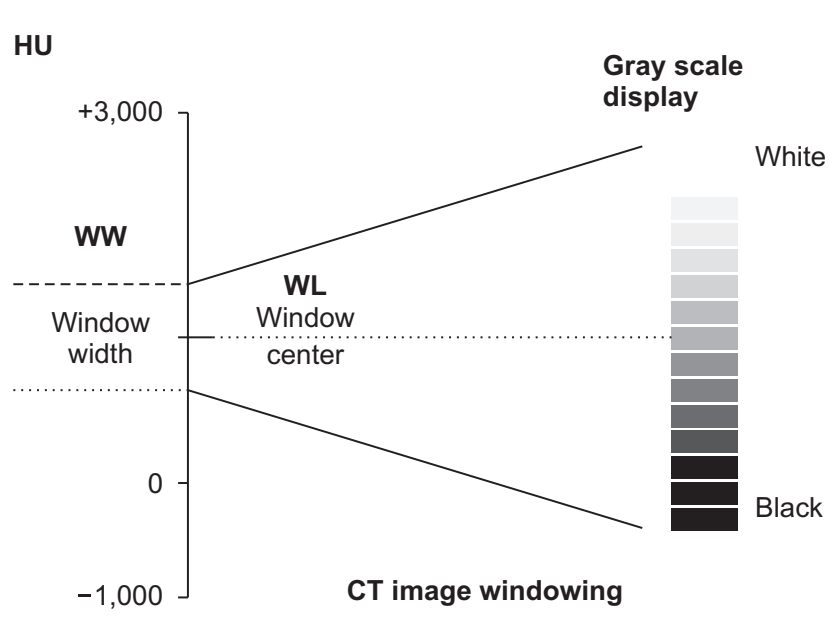

b

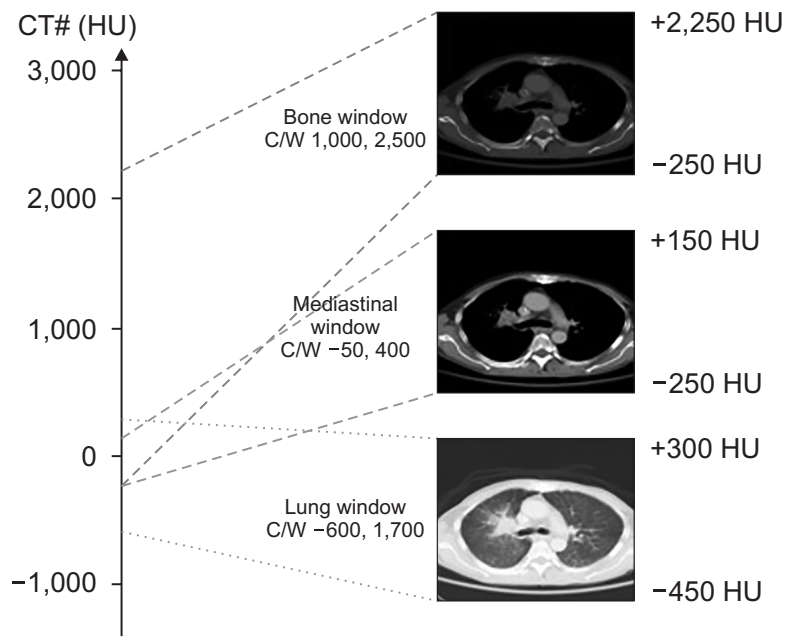

Fig. 8. Computed tomography (CT) image windowing (a), chest CT scan displayed according to the adjustment of bone, mediastinal, and lung windows (b). HU, Hounsfield unit; WW, window width; WL, window level.
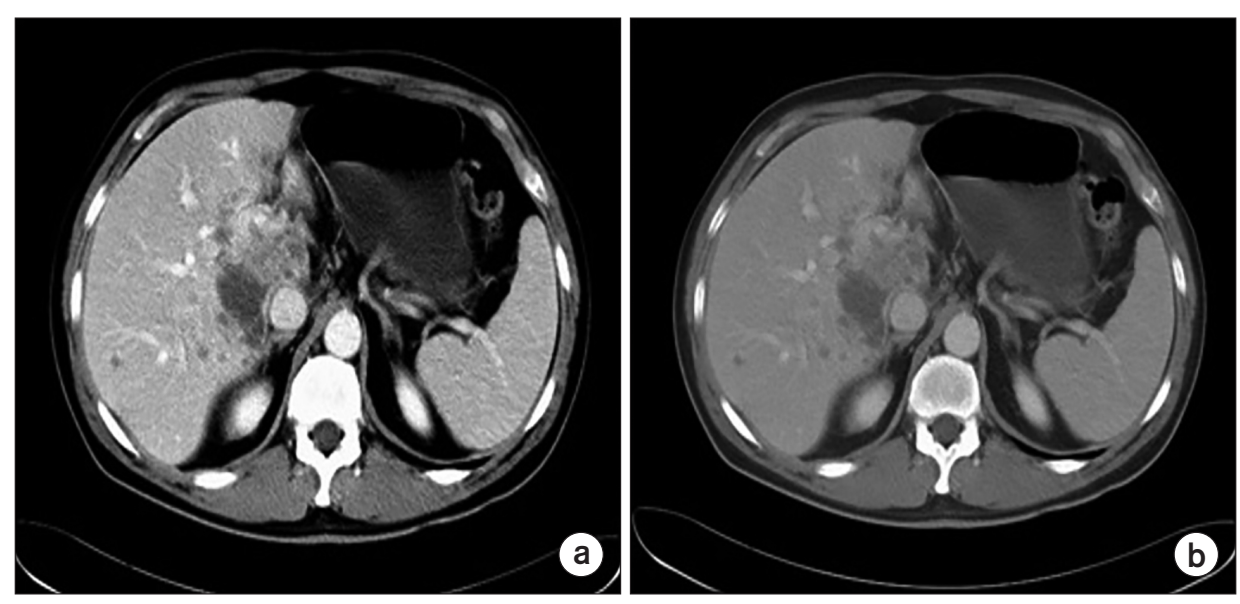

Fig. 9. Computed tomography images shown in Fig. 8 above indicate how the adjustment of the window width changes the contrast of the image, narrow window width (a) and wide window width (b).

can be used to visualize the vessels of the heart, aorta, and other large blood vessels, lungs, kidneys, head and neck, and arms and legs. CTA images can be $3 \mathrm{D}$ reconstructed so that the cerebral vessels and accompanying pathology can be rotated and viewed from all angles. Contrast media are used to image structures that are not normally visible, or are not visible very distinctly. Intravenous contrast agents are used to enhance organs and visualize blood vessels. Oral contrast agents are used to visualize the digestive tract. The contrast media for CTA is injected through an intravenous line in the arm or hand. In general, CT contrast media is a colorless and transparent iodine compound, that is, a sub- stance that X-rays cannot penetrate. In one CT exam, the amount of approximately $100-150 \mathrm{~mL}$ is injected into a vein at the rate of $2-3 \mathrm{~mL} / \mathrm{s}$ with a CT contrast injector. The contrast media can lead to adverse reactions such as nausea, vomiting, and/or hives, uncommon allergic reactions, brief loss of consciousness, bronchospasm, kidney damage, and others.

Before an external beam radiotherapy treatment planning process, the radiation oncologists plan to determine the type of cancer, its exact location, the size and shape of the cancer, the cancer staging, the critical structures closely located to the cancer, the radiation dose needed in the can- 
cer treatment, and the patient's general health and medical history.

CT simulators (CTSim) dedicated to radiotherapy are based on general diagnostic CT scanners, with few additional modifications. CTSim typically include a laser positioning and marking system as a reference for patient positioning, a larger bore size to accommodate patient immobilization devices, a flat tabletop to replicate the radiotherapy treatment unit couch, and a virtual simulation software workstation. The CTsim 3D imaging data of the patient's anatomy provide various information for more accurate delineation of the tumor and the surrounding normal tissues. In addition, the pixel HU values inherently include the associated tissue density information used to compute radiation dose distributions, which is a necessity for $3 \mathrm{D}$ radiotherapy treatment planning for X-ray radiation treatments. The conversion is accomplished with a phantom calibration, and annual checks are required. State-ofthe-art CTSim are also capable of acquiring 4D imaging data. The 4D CT simulations are applicable for the internal organs affected by respiratory motion. These advanced CT imaging simulation techniques allow accurate tumor localization, treatment visualization, and subsequent treatment delivery [34].

MicroCT is similar in terms of the imaging acquisition principles to common diagnostic CTs but on a much smaller bore-size scale and applied slice thickness, and with a greatly increased spatial resolution. The common microCT scanners can image objects (as large as up to 20 $\mathrm{cm}$ in diameter) with pixel sizes in the micrometer range. The microCT scanner can be used to study the internal structure of both material and biological samples for materials science and small animal imaging, such as mice and rats, and in some cases rabbits, without the need to section the samples, thus preserving the samples or specimens for future studies. Typical microCT scanners are typically used for small animals (in vivo scanners), biomedical samples, food, microfossils, and other studies where minute detail is desired. They are equipped with a detection system for a sealed microfocus X-ray source and CCD (charge-coupled device) camera, which are operated on a table top with high-accelerating voltages which vary between 70 and 225 $\mathrm{kV}$ with specific beam currents, a few tens of Watts of pow- er, aluminum filters, radiation doses less than few $\mu \mathrm{Sv} / \mathrm{h}$ at $10 \mathrm{~cm}$ from the instrument's surface, long scanning times ranging from a few min up to 10 hours depending on the size of the region of interest, and an image reconstruction time of a few minutes.

CBCT has become increasingly important in radiation treatment planning, dedicated breast imaging, diagnosis in implant dentistry in medical fields, and industrial applications as a nondestructive testing tool. The fundamental difference between CBCT and conventional helical CT is that CBCT utilizes a divergent cone-shaped X-ray beam and a large area flat panel detector compared with a narrow collimated, fan-shaped X-ray beam and a linear group of detectors used in helical CT. CBCT captures a full image volume in a single rotation around a stationary object and uses $3 \mathrm{D}$ volume reconstruction algorithms. The use of CBCT in radiotherapy is increasing owing to the widespread implementation of $\mathrm{kV}$ systems mounted on the currently available linear accelerators. The primary use of gantry-mounted kV CBCT is for image-guided radiotherapy to verify patient positioning and setup, localization of tumors, and tracking of tumor changes during the course of treatment. However, it also opens up the possibility of realtime re-optimization of treatment plans for adaptive radiotherapy as HU values are obtained and can be calibrated for use in treatment planning. The use of CBCT in radiotherapy with 500-1,000 projections and acquisition times spanning $40 \mathrm{~s}$ to 2 minutes per gantry rotation cause poor image quality of the moving object within all slices and scattered radiation owing to the conical-shaped beam. Additionally, cone beam geometry covers a large FOV within one rotation, which contributes to a larger scattered radiation component compared with fan-beam helical CT. However, the use of CBCT requires additional treatment time and radiation dose for clinical applications. These have negative impacts on physicians/residents who have to be present at the time of the CBCT acquisition because offline viewing is not available. CBCT imaging doses in radiation therapy has traditionally been ignored owing to its low magnitude and frequency in comparison with the therapeutic dose used to treat patients. CBCT increases the dose delivered not only to the tumor but also to the healthy tissues. The additional dose delivered to patients can amount to 2-8 cGy depend- 
ing on the treatments [35-43].

Portable (or mobile) CT is another clinical CT type. Its main characteristics are capable of moving from any room in the hospital into an advanced imaging suite, including the operating room, intensive care unit (ICU), radiation oncology suites, and the emergency department, needing versatile real-time portable imaging at the patient's bedside. These portable CTs are specialized for the patients to improve the quality of care, and to ameliorate the difficulties associated with the transportation of patients to the conventional CT imaging scanner when they are in the ICU, clinics, operating room, and the emergency department. The portable CT scanning mainly includes neuroradiological head and neck imaging in ICU, interoperative imaging of the bone and sinuses in the ENT, and trauma, orthopedic, and vascular imaging during surgery, etc. [44,45].

Low-dose CT (LDCT) examinations for lung cancer screenings are currently employed to detect lung nodules that may represent lung cancer, and to find treatable lung cancer cases using special image acquisition protocols with a substantially lower radiation dose offered by most modern MDCT scanners. The most important scan parameters for optimizing radiation dose from LDCT are the tube current, low-tube potentials which range from 80 to $100 \mathrm{kV}$, and novel low-dose reconstruction algorithm, which results in up to five times lower radiation exposure in a LDCT scan than that absorbed during a standard CT scan. LDCT screening is known to be a much more effective process than general chest X-ray radiography. LDCT scans are generally used only for adults aged 55 to 80 years who have a history of smoking and who are still smoking or have quit smoking during the past 15 years, or on patients who already have lung cancer as the margin of error is usually too great with people who have no signs or symptoms of the disease, often resulting in more standard tests than necessary [46-51].

\section{Radiation dose of computed tomography}

The radiation dose received from CT examinations depends on the type of the CT scanner and examination, the size of the body part examined, and the image acquisition parameters (such as $\mathrm{kVp}, \mathrm{mA}$, exposure time, $\mathrm{X}$-ray beam collimation, scan pitch, dose reduction options, etc.). Common radiation doses by CT scans for abdominal scanning conditions of $120 \mathrm{kV}, 200 \mathrm{~mA}, 2$ seconds, length slice: 100 $\mathrm{mm}$, phantom with $\phi 320 \mathrm{~mm}$, were $21 \mathrm{mGy}$ at the center of phantom, and $35 \mathrm{mGy}$ at the periphery of the phantom. The effective doses from CT diagnostic procedures are typically estimated to be in the range of 1 to $10 \mathrm{mSv}$. Table 1 lists representative CT diagnostic procedures and associated radiation doses [52-57].

\section{Quality control and imaging phantom of computed tomography}

The periodic quality control (QC) (quality assurance) of CT is important to maintain proper performance of electromechanical components, optimum image quality, and reasonable radiation doses to the patients. The CT QC program is classified as the roles and responsibilities of the radiologist, radiologic technologist, and medical physicist, and the routine test cycle of weekly tests (some perform daily), monthly tests, semi-annual tests, and annual tests. The ACR recommended in the CT QC manual the minimum QC test frequencies, such as the annual tests of qualified medical physicist surveys, including participation in the review of clinical protocols with the CT protocol and management team, scout prescription and alignment light accuracy, table travel accuracy, radiation beam width, low-contrast performance, spatial resolution, CT number accuracy, artifact evaluation, CT number uniformity, dosimetry, CT scanner display calibration, radiological technologist QC tests of the water's CT number and standard deviation (daily), artifact

Table 1. Typical effective radiation dose values for common computed tomography (CT) examinations

\begin{tabular}{lc}
\hline Diagnostic procedure & Typical effective dose $(\mathrm{mSv})$ \\
\hline Head CT & $1-2$ \\
Chest CT & $5-7$ \\
Abdomen CT & $5-7$ \\
Abdomen and pelvis CT & $8-14$ \\
Coronary CT angiography & $5-15$ \\
Coronary calcium CT & $1-3$ \\
\hline
\end{tabular}

Data from AAPM Report No. 96: The Measurement, Reporting, and Management of Radiation Dose in CT. American Association of Physicists in Medicine (AAPM) Task Group 23 (2008). 

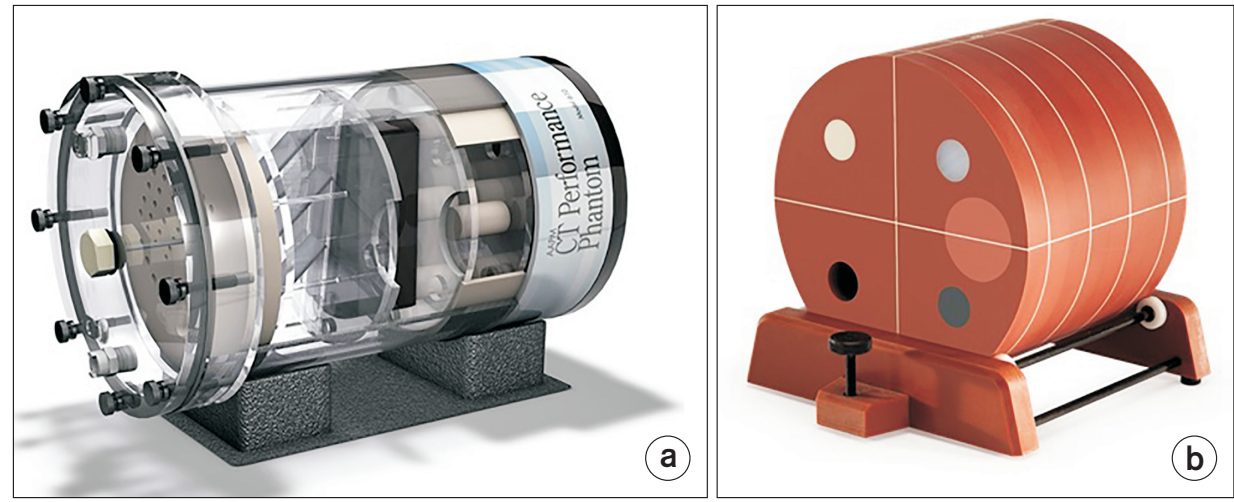

Fig. 10. (a) American Association of Physicists in Medicine CT performance phantom model 76-410. (b) American College of Radiology CT 464 phantom designed to examine a broad range of image quality parameters.

evaluation (daily), wet laser printer QC (weekly), visual checklist (monthly), dry laser printer QC (monthly), and gray level performance of CT scanner acquisition display monitors (monthly). The CT performance phantoms used to CT image acceptance testing and QC are shown in Fig. 10 [58-64].

\section{Conclusions}

The understanding of CT characteristics will provide more effective and accurate patient care in the fields of diagnostics and radiotherapy, and can lead to the improvement of image quality and the optimization of the exposed doses.

\section{Acknowledgements}

I would like to express my appreciation to my son, SungJin Jung, who reviewed and encouraged me to prepare this manuscript. The research was supported by the FutureChem Co., Ltd and Division of Applied RI, Korean Institute of Radiological \& Medical Sciences (KIRAMS).

\section{Conflicts of Interest}

The authors have nothing to disclose.

\section{Availability of Data and Materials}

All relevant data are within the paper and its Supporting
Information files.

\section{References}

1. Goodman PC. The new light: discovery and introduction of the X-ray. AJR Am J Roentgenol. 1995;165:1041-1045.

2. Mould RF. Invited review: Röntgen and the discovery of Xrays. Br J Radiol. 1995;68:1145-1176.

3. Beatty J. The radon transform and the mathematics of medical imaging. Waterville: Colby College, 2012 [cited $2021 \mathrm{Feb}$ 15]. Available from: https://digitalcommons.colby. edu/cgi/viewcontent.cgi?article=1649\&context=honorsthe ses.

4. Barrett HH. III The radon transform and its applications. Prog Opt. 1984;21:217-286.

5. Hornegger J, Maier A, Kowarschik M, CT Image reconstruction basics. Radiology Key, 2018 [cited 2021 Feb 15]. Available from: https://radiologykey.com/ct-image-reconstruction-basics/.

6. Maier A, Steidl S, Christlein V, Hornegger J. Medical imaging systems: an introductory guide. Cham: Springer Nature Switzerland AG; 2018:148-167.

7. Goldman LW. Principles of CT and CT technology. J Nucl Med Technol. 2007;35:115-128; quiz 129-130.

8. Bae KT, Whiting BR. Basic principles of computed tomography physics and technical considerations. Radiology Key, 2016 [cited 2021 Feb 15]. Available from: https://radiologykey.com/basic-principles-of-computed-tomographyphysics-and-technical-considerations/.

9. Hounsfield GN. Computerized transverse axial scanning 
(tomography). 1. Description of system. Br J Radiol. 1973;46: 1016-1022.

10. Rincon-Guio C, Gómez AM, Charry JD. The role of computed tomography as a prognostic tool in traumatic brain trauma. Imaging Med. 2017;9:171-178.

11. Kachelriess M, Schaller S, Kalender WA. Advanced singleslice rebinning in cone-beam spiral CT. Med Phys. 2000; 27:754-772.

12. Kak AC, Slaney M. Principles of computerized tomographic imaging. Philadelphia: Society of Industrial and Applied Mathematics; 1987:49-112.

13. Stierstorfer K, Rauscher A, Boese J, Bruder H, Schaller S, Flohr T. Weighted FBP--a simple approximate 3D FBP algorithm for multislice spiral CT with good dose usage for arbitrary pitch. Phys Med Biol. 2004;49:2209-2218.

14. Flohr TG, Schaller S, Stierstorfer K, Bruder H, Ohnesorge BM, Schoepf UJ. Multi-detector row CT systems and imagereconstruction techniques. Radiology. 2005;235:756-773.

15. Hata A, Yanagawa M, Honda O, Kikuchi N, Miyata T, Tsukagoshi S, et al. Effect of matrix size on the image quality of ultra-high-resolution CT of the lung: comparison of $512 \times 512,1024 \times 1024$, and $2048 \times 2048$. Acad Radiol. 2018;25: 869-876.

16. Ginat DT, Gupta R. Advances in computed tomography imaging technology. Annu Rev Biomed Eng. 2014;16:431-453.

17. Garvey CJ, Hanlon R. Computed tomography in clinical practice. BMJ. 2002;324:1077-1080.

18. Flohr T. CT systems. Curr Radiol Rep. 2013;1:52-63.

19. van Ooijen PM, van Geuns RJ, Rensing BJ, Bongaerts AH, de Feyter PJ, Oudkerk M. Noninvasive coronary imaging using electron beam CT: surface rendering versus volume rendering. AJR Am J Roentgenol. 2003;180:223-226.

20. Kopp AF, Klingenbeck-Regn K, Heuschmid M, Küttner A, Ohnesorge B, Flohr T, et al. Multislice computed tomography: basic principles and clinical applications. Electromedica. 2000;68:94-105.

21. Grajo JR, Patino M, Prochowski A, Sahani DV. Dual energy CT in practice: basic principles and applications. Appl Radiol. 2016;45:6-12.

22. Sajja S, Lee Y, Eriksson M, Nordström H, Sahgal A, Hashemi M, et al. Technical principles of dual-energy cone beam computed tomography and clinical applications for radiation therapy. Adv Radiat Oncol. 2019;5:1-16.
23. Ohana M, Jeung MY, Labani A, El Ghannudi S, Roy C. Thoracic dual energy CT: acquisition protocols, current applications and future developments. Diagn Interv Imaging. 2014;95:1017-1026.

24. Coursey CA, Nelson RC, Boll DT, Paulson EK, Ho LM, Neville AM, et al. Dual-energy multidetector CT: how does it work, what can it tell us, and when can we use it in abdominopelvic imaging? Radiographics. 2010;30:1037-1055.

25. Rassouli N, Etesami M, Dhanantwari A, Rajiah P. Detectorbased spectral CT with a novel dual-layer technology: principles and applications. Insights Imaging. 2017;8:589-598.

26. Silva AC, Morse BG, Hara AK, Paden RG, Hongo N, Pavlicek W. Dual-energy (spectral) CT: applications in abdominal imaging. Radiographics. 2011;31:1031-1046; discussion 1047-1050.

27. Lee SC, Kim HK, Chun IK, Cho MH, Cho MH, Lee SY. Development of a micro-CT system for small animal imaging. J Biomed Eng Res. 2004;25:97-102.

28. Nam KY, Kim KW, Kim JH, Son HH, Ryu JH, Kang SH, et al. Micro-CT system for small animal imaging. Korean J Med Phys. 2008;19:102-112.

29. Goldman LW. Principles of CT: radiation dose and image quality. J Nucl Med Technol. 2007;35:213-225; quiz 226-228.

30. Calhoun PS, Kuszyk BS, Heath DG, Carley JC, Fishman EK. Three-dimensional volume rendering of spiral CT data: theory and method. Radiographics. 1999;19:745-764.

31. Duran AH, Duran MN, Masood I, Maciolek LM, Hussain H. The additional diagnostic value of the three-dimensional volume rendering imaging in routine radiology practice. Cureus. 2019;11:e5579.

32. Grunert P, Müller-Forell W, Darabi K, Reisch R, Busert C, Hopf N, et al. Basic principles and clinical applications of neuronavigation and intraoperative computed tomography. Comput Aided Surg. 1998;3:166-173.

33. Liguori C, Frauenfelder G, Massaroni C, Saccomandi P, Giurazza F, Pitocco F, et al. Emerging clinical applications of computed tomography. Med Devices (Auckl). 2015;8:265278.

34. Davis AT, Palmer AL, Nisbet A. Can CT scan protocols used for radiotherapy treatment planning be adjusted to optimize image quality and patient dose? A systematic review. Br J Radiol. 2017;90:20160406.

35. Posiewnik M, Piotrowski T. A review of cone-beam CT ap- 
plications for adaptive radiotherapy of prostate cancer. Phys Med. 2019;59:13-21.

36. Srinivasan K, Mohammadi M, Shepherd J. Applications of linac-mounted kilovoltage Cone-beam Computed Tomography in modern radiation therapy: a review. Pol J Radiol. 2014;79:181-193.

37. Abramovitch K, Rice DD. Basic principles of cone beam computed tomography. Dent Clin North Am. 2014;58:463484.

38. Scarfe WC, Farman AG. What is cone-beam CT and how does it work? Dent Clin North Am. 2008;52:707-730, v.

39. Kumar M, Shanavas M, Sidappa A, Kiran M. Cone beam computed tomography - know its secrets. J Int Oral Health. 2015;7:64-68.

40. Srinivasan K, Mohammadi M, Shepherd J. Cone beam computed tomography for adaptive radiotherapy treatment planning. J Med Biol Eng. 2014;34:377-385.

41. Miracle AC, Mukherji SK. Conebeam CT of the head and neck, part 1: physical principles. AJNR Am J Neuroradiol. 2009;30:1088-1095.

42. Sepulcri M, Paronetto C, El Khouzai B, Novo A, Aldegheri $\mathrm{V}$, Scaggion A, et al. Effectiveness of cone beam computed tomography imaging during radiation therapy for the detection of initial coronavirus lung disease 2019. Adv Radiat Oncol. 2020;5:697-699.

43. Guckenberger M. Image-guided radiotherapy based on kilovoltage cone-beam computed tomography - a review of technology and clinical outcome. Eur Oncol Haematol. 2011;7:121-124.

44. Maher MM, Hahn PF, Gervais DA, Seoighe B, Ravenscroft JB, Mueller PR. Portable abdominal CT: analysis of quality and clinical impact in more than 100 consecutive cases. AJR Am J Roentgenol. 2004;183:663-670.

45. Rumboldt Z, Huda W, All JW. Review of portable CT with assessment of a dedicated head CT scanner. AJNR Am J Neuroradiol. 2009;30:1630-1636.

46. Niu T, Zhu L. Low-dose quantitative cone-beam CT imaging in radiation therapy. Paper presented at: 2012 IEEE Nuclear Science Symposium and Medical Imaging Conference Record (NSS/MIC); 2012 Oct 27-Nov 3; Anaheim, USA. p. 2907-2909.

47. Larke FJ, Kruger RL, Cagnon CH, Flynn MJ, McNitt-Gray $\mathrm{MM}, \mathrm{Wu} \mathrm{X}$, et al. Estimated radiation dose associated with low-dose chest CT of average-size participants in the National Lung Screening Trial. AJR Am J Roentgenol. 2011;197: 1165-1169.

48. Ono K, Hiraoka T, Ono A, Komatsu E, Shigenaga T, Takaki $\mathrm{H}$, et al. Low-dose CT scan screening for lung cancer: comparison of images and radiation doses between low-dose CT and follow-up standard diagnostic CT. Springerplus. 2013;2:393.

49. Su AW, Hillen TJ, Eutsler EP, Bedi A, Ross JR, Larson CM, et al. Low-dose computed tomography reduces radiation exposure by $90 \%$ compared with traditional computed tomography among patients undergoing hip-preservation surgery. Arthroscopy. 2019;35:1385-1392.

50. Rampinelli C, Origgi D, Bellomi M. Low-dose CT: technique, reading methods and image interpretation. Cancer Imaging. 2013;12:548-556.

51. Kubo T, Ohno Y, Takenaka D, Nishino M, Gautam S, Sugimura K, et al. Standard-dose vs. low-dose CT protocols in the evaluation of localized lung lesions: capability for lesion characterization-iLEAD study. Eur J Radiol Open. 2016;3:67-73.

52. Costello JE, Cecava ND, Tucker JE, Bau JL. CT radiation dose: current controversies and dose reduction strategies. AJR Am J Roentgenol. 2013;201:1283-1290.

53. Willekens I, Buls N, Lahoutte T, Baeyens L, Vanhove C, Caveliers V, et al. Evaluation of the radiation dose in microCT with optimization of the scan protocol. Contrast Media Mol Imaging. 2010;5:201-207.

54. Lell MM, Wildberger JE, Alkadhi H, Damilakis J, Kachelriess $\mathrm{M}$. Evolution in computed tomography: the battle for speed and dose. Invest Radiol. 2015;50:629-644.

55. Smith-Bindman R, Lipson J, Marcus R, Kim KP, Mahesh M, Gould R, et al. Radiation dose associated with common computed tomography examinations and the associated lifetime attributable risk of cancer. Arch Intern Med. 2009; 169:2078-2086.

56. Obenaus A, Smith A. Radiation dose in rodent tissues during micro-CT imaging. J X-Ray Sci Technol. 2004;12:241249.

57. Goo HW. CT radiation dose optimization and estimation: an update for radiologists. Korean J Radiol. 2012;13:1-11.

58. American Association of Physicists in Medicine. Specification and acceptance testing of computed tomography 
scanners. Alexandria: American Association of Physicists in Medicine. 1993; 39.

59. An HJ, Son JM, Jin HM, Sung JW, Chun MS. Acceptance test and clinical commissioning of CT simulator. Prog Med Phys. 2019;30:160-166.

60. American College of Radiology. The 2017 Computed Tomography Quality Control manual. American College of Radiology; 2017.

61. Mutic S, Palta JR, Butker EK, Das IJ, Huq MS, Loo LN, et al. Quality assurance for computed-tomography simulators and the computed-tomography-simulation process: report of the AAPM Radiation Therapy Committee Task Group No. 66. Med Phys. 2003;30:2762-2792.

62. Appel E, Kröpil P, Bethge OT, Aissa J, Thomas C, Antoch
G, et al. Quality assurance in CT: implementation of the updated national diagnostic reference levels using an automated CT dose monitoring system. Clin Radiol. 2018;73:677. e13-e677.e20.

63. Mansour Z, Mokhtar A, Sarhan A, Ahmed MT, El-Diasty T. Quality control of CT image using American College of Radiology (ACR) phantom. Egypt J Radiol Nucl Med. 2016;47: 1665-1671.

64. McCollough CH, Bruesewitz MR, McNitt-Gray MF, Bush K, Ruckdeschel T, Payne JT, et al. The phantom portion of the American College of Radiology (ACR) computed tomography (CT) accreditation program: practical tips, artifact examples, and pitfalls to avoid. Med Phys. 2004;31:2423-2442. 